cond-mat.mtrl-sci

\title{
Calibration of oscillation amplitude in Dynamic Scanning Force Microscopy
}

Juan Francisco González Martínez, ${ }^{*}$ Inés Nieto-Carvajal, ${ }^{\dagger}$ and Jaime Colchero Paetz ${ }^{\ddagger}$

(Dated: October 29, 2018)

\begin{abstract}
A method to precisely calibrate the oscillation amplitude in Dynamic Scanning Force Microscopy (DSFM) is described. It is experimentally shown that a typical electronics used to process the dynamic motion of the cantilever can be adjusted to transfer the thermal noise of the cantilever motion from its resonance frequency to a much lower frequency within the typical bandwidth of the corresponding electronics. Therefore, the thermal noise measured in the in-phase ("phase") and out-of-phase ("amplitude") output of such an electronics can be related to the thermal energy $k T$. If the force constant of the cantilever is known then the oscillation amplitude can be precisely calibrated from the thermal power measured in these signals. Based on this concept, two procedures for the calibration of the oscillation amplitude are proposed. One is based on simple calculation of the Root Mean Square (RMS) measured at the outputs of the electronics used to process the dynamic motion of the cantilever, and the other one is based on analysis of the corresponding spectrum and the calculation of the quality factor, the resonance frequency and the signal strength.
\end{abstract}

PACS numbers: 07.79.Lh

Keywords: Dynamic Scanning Force Microscopy, oscillation amplitude calibration, normal force calibration, force constant, optical detector sensitivity.

\footnotetext{
*jfgm@um.es

† inc2@um.es

$\ddagger$ colchero@um.es
} 


\section{INTRODUCTION}

Scanning Force Microscopy (SFM) is an extremely versatile tool for Nanoscience that has a very high resolution, but usually only a modest precision. When SFM is used as a microscope, precise calibration of piezo movements is necessary, as well as careful control of piezo hysteresis and non-linearities. When used as a force measuring instrument a precise calibration of the displacement detector as well as the force constant of the cantilever is required. Dynamic SFM (DSFM) is by now probably the most extended SFM mode, since it allows operation at very low forces, and operates in the non-contact regime [1-4]. In DSFM the frequency (or phase) of the tip-sample system and the oscillation amplitude are the basic signals that are measured and should thus be calibrated for precise measurements. Precise measurement of frequency is easy and is implemented directly in most instruments. Calibration of oscillation amplitude is an issue that has received little attention, and less than what we believe it deserves. In Frequency Modulation DSFM (FM-DSFM) the frequency shift is related to (conservative) tip-sample interaction and the oscillation amplitude to dissipation. For Amplitude Modulation (AM-DSFM or "tapping mode") this separation is less evident, nevertheless the oscillation amplitude is an important parameter, since it critically determines non-linearity of tip-sample interaction [5, 6], onset of bi-stability and chaos [7-9] as well as the precise interaction regime (attractive vs. repulsive) used for imaging [10]. Finally, as shown recently, if correctly calibrated the oscillation amplitude can be used to obtain a reliable image of the "true topography" that is independent of feedback parameters and scanning speed [11]. In the present work we will discuss a method to precisely calibrate the oscillation amplitude in DSFM. Essentially, the method relates the thermal noise measured in the "amplitude" and "phase" outputs of the electronics to the thermal energy $k T$. If the force constant of the cantilever is known then the oscillation amplitude can be calibrated from the thermal power measured in these signals.

\section{CALIBRATION PROCEDURES}

In the present work we discuss the response of the DSFM Dynamic Unit (DSFM-DU) in the frequency domain because we will analyze the response of the system to thermal noise $z_{t h}(t)$. Unfortunately, thermal noise is non-trivial to describe using a "coherent" superposition of harmonic signals, as it is implicitly assumed in the Fourier Transform $z(t)=\int d \nu z(\nu) e^{i 2 \pi \nu t}$. The correct description of noise in the time domain should involve wavelet transforms [12], which is however beyond of the scope of the present work. We note that in the context of the present work the 
DSFM-DU is used in a non-standard way, where no oscillation is applied to excite the cantilever, and the reference oscillator frequency $\nu_{r}$ is not at the resonant frequency of the cantilever. Instead, the DSFM-DU is adjusted so that $\nu_{r} \approx \nu_{0}-b w / 2$ where $b w=1 / \tau(\tau$ : time constant) is the bandwidth of the DSFM-DU. As discussed in detail in Appendix A. The thermal peak of the cantilever appears centered within the spectral range of the output of the DSFM-DU.

Essentially, the reason for this frequency shift is that when the normal force signal is internally multiplied with the reference frequency $\nu_{r}$ of the DSFM-DU, frequency components at $\nu_{0}-\nu_{r}$ and $\nu_{0}+\nu_{r}$ appear (for example from terms like $\cos \left(2 \pi \nu_{0} t\right) \cos \left(2 \pi \nu_{r} t\right)$, see also figure 1 for a graphical representation). Usually, the DSFM-DU is opperated with $\nu_{0}=\nu_{r}$, then thermal noise is observed at very low frequencies $\nu \approx 0$, that is, at DC. At this low frequency the thermal noise is usually not clearly distinguised from pink noise $(1 / \nu$-noise) of typical electronic components. However, when $\nu_{0} \neq \nu_{r}$, the thermal peak is shifted to the difference frequency $\nu_{0}-\nu_{r}$, which may be well below the resonance frequency, $\nu_{0}$. As discussed above, we typically adjust $\nu_{r}$ so that $\nu_{0}-\nu_{r} \approx b w / 2 \approx 4 \mathrm{kHz}$ in our system.

Figure 2 shows experimental data for the deflection signal $u_{d}(t)$, and the output $u_{y}(t)$ measured when the DSFM-DU is configured as just discussed. The main graphs show spectra, and the insets time domain data. The spectra have been acquired using a Spectrum Analyzer [13] as well as the "noise" function of a commercial Lock-In Amplifier [14]. The lines through the data points correspond to a "Lorentzian + offset" fit function $f(\nu)[15,16]$,

$$
f(\nu)=\frac{e_{t h}^{2}}{\left(1-\left(\nu / \nu_{0}\right)^{2}\right)^{2}+\left(\nu /\left(\nu_{0} Q\right)\right)^{2}}+e_{n}^{2}=e_{t h}^{2}|g(\nu)|^{2}+e_{n}^{2}
$$

where $g(\nu)$ is the mechanical gain of the cantilever (see relation (A1)). The corresponding parameters for the different fits are specified in table I, and discussed in more detail below. The scattered points around the horizontal lines show the error of the experimental power spectrum to the corresponding fit, which show little tendency for both spectra, therefore we conclude that the chosen fit functions describe well the experimental data. Both spectra lead to essentially the same results, in particular for the values of the resonance frequency $\nu_{0}$ and the quality factor $Q$. Since the DSFM-DU internally amplifies the normal force signal, the signal strength of the deflection signal $u_{d}(t)$ is smaller than that of the DSFM-DU outputs $u_{x}(t)$ and $u_{y}(t)$, leading to different thermal noise $e_{t h}$ and electronic noise $e_{n}$ for the deflection spectrum $u_{d}(\nu)$ compared to $u_{x}(\nu)$ and $u_{y}(\nu)$. We therefore conclude that the outputs $u_{x}(t)$ and $u_{y}(t)$ of the DSFM-DU essentially reproduce the thermal noise spectrum of the deflection signal and that the DSFM-DU therefore "sees" (internally amplified) thermal noise shifted to the frequency $\nu_{0}-\nu_{r}$. It should be therefore possible to apply the well-known thermal calibration method based on the equipartition theorem 
[16-22],

$$
\frac{c}{2}\left\langle z_{t h}^{2}(t)\right\rangle_{\mathrm{rms}}=\frac{1}{2} k T
$$

to the calibration of the oscillation amplitude.

We note that, as for the case of normal force calibration, relation (2) has implicitly two unknowns: the force constant $c$ of the cantilever and a sensitivity calibration $\beta$ (units: $\mathrm{nm} / \mathrm{V}$ ) to convert the measured signal $u(t)$ (Volts) into physical length units (Nanometers):

$$
z(t)=\beta u(t)
$$

In the present work, we will assume that the force constant is known or has been determined independently (see, for example [22, 23]), and will thus use (2) to calibrate the sensitivity $\beta$ of the DSFM-DU. We will present and discuss two procedures based on equation (2): a simple one which only requires estimation of the RMS noise of the output of the DSFM-DU, and a second one taking into account the full spectral response of the outputs $u_{x}(t)$ and $u_{y}(t)$.

\section{A. Calibration based on root mean square estimation}

The first calibration procedure is implemented experimentally by acquiring the outputs $u_{x}(t)$ and $u_{y}(t)$ for a certain period of time with the laser on and with the laser off. The noise power that is related to thermal noise is then,

$$
\left(u_{t h}\right)^{2}=\left\langle u_{\mathrm{on}}^{2}(t)\right\rangle_{\mathrm{rms}}-\left\langle u_{\mathrm{off}}^{2}(t)\right\rangle_{\mathrm{rms}}
$$

The reason to acquire data with the laser off is to estimate and subtract electronic noise that is not related to the thermal noise of the cantilever motion. In particular for hard cantilevers, where thermal noise is quite small (see below) the calibration may be severely wrong if this electronic noise is not subtracted. Without substraction, it is implicitly assumed that all measured noise (that is, also electronic noise) is thermal noise. The voltage noise $\Delta u_{t h}^{x}=\sqrt{\left\langle\left(u_{t h}^{x}\right)^{2}\right\rangle}$ of the output $u_{x}(t)$ is related to the "phase" noise while the noise $\Delta u_{t h}^{y}=\sqrt{\left\langle\left(u_{t h}^{y}\right)^{2}\right\rangle}$ is related to the oscillation amplitude. Relation (4) can of course also be applied to the fluctuations measured in the deflection signal $u_{d}(t)$, then $\Delta u_{t h}^{d}=\sqrt{\left\langle\left(u_{t h}^{d}\right)^{2}\right\rangle}$ is the deflection noise (in Volts) of the cantilever motion. We recall that thermal noise is distributed equally between the in-phase and out-of-phase components; then, if the amplification of the outputs $u_{x}(t)$ and $u_{y}(t)$ as well as the deflection $u_{d}(t)$ are the same (the latter is usually not the case, see below),

$$
\Delta u_{t h}^{x}=\Delta u_{t h}^{y}=\Delta u_{t h}^{d} / \sqrt{2}
$$


since the total deflection signal and the in-phase and out-out-phase componentes $x$ and $y$ are related by $u_{d}^{2}(t)=u_{x}^{2}(t)+u_{y}^{2}(t)$ and therefore $\beta^{x}=\beta^{y}$ (see, for example, [15]). The values $\Delta u_{t h}^{x}$ and $\Delta u_{t h}^{y}$ can be either computed directly from the time domain signals $u_{x}(t)$ and $u_{y}(t)$, or by adding (integrating) the noise spectrum in the frequency domain. Due to the different normalizations found for the Fourier Transform and the Power Spectral Density in the literature as well as in software packets, it is usually more secure to use the first option. The insets presented in figure 2 show the data used for this calibration procedure. The data from the DSFM-DU is presented as scatter plot $\left(u_{x}(t), u_{y}(t)\right)$; as it would be visualized with an oscilloscope in $x-y$ mode. Data is shown for the laser on and off. The diameter of this scatter plot is proportional to the fluctuations in both directions. The data corresponding to the deflection signal $u_{d}(t)$ is shown "normally" as a function of time, again data is shown for the laser on and off. Calibration factors are computed directly as $\beta^{y}=z_{t h} / \Delta u_{t h}^{y} / \sqrt{2}$ (see relation (5) for the factor $\sqrt{2}$ ) and $\beta^{d}=z_{t h} / \Delta u_{t h}^{d}$ with $z_{t h}=\sqrt{k T / c}$ rms thermal noise movement (unit: nm, from equation (2)). The results obtained with this first method are summarized in the left column of table I (termed "first procedure"). The calibration factors for the cantilever deflection and for the (amplitude) output of the DSFM-DU have been measured for a cantilever with $c=1.6 \mathrm{~N} / \mathrm{m}$, as determined using Sader's method [23] from the resonance frequency and quality factor (see table I).

\section{B. Calibration using the thermal noise spectrum}

The second calibration procedure is based on estimating the area under the thermal noise spectrum using the values determined from the fit to a "Lorentzian function + electronic noise function", $f(\nu)$ from relation (1). In this context we recall that $\int_{0}^{\infty} d \nu|g(\nu)|^{2}=Q \nu_{0} \pi / 2$, therefore the measured "thermal noise power" as determined from the fit parameters is $e_{t h}^{2} Q \nu_{0} \pi / 2$, where $e_{t h}^{2}$ is the strength of the thermal signal (units: $\mathrm{V}^{2} / \mathrm{Hz}$ ). As discussed previously, in the frequency domain the DSFM-DU shifts the original deflection signal $z(\nu)$ from a signal centered around the resonance frequency $\nu_{0}$ to signals $u_{x}(\nu)$ and $u_{y}(\nu)$ centered at $\nu_{0}-\nu_{r} \approx b w / 2$ where $\nu_{r}$ is the reference frequency adjusted by the DSFM-DU. In addition, the original signal $u_{d}(t)$ is amplified by the internal gains of the DSFM-DU. In principle, there are two possible ways of processing the data obtained from the DSFM-DU: the spectra $\left|u_{x}(\nu)\right|^{2}$ and $\left|u_{y}(\nu)\right|^{2}$ may be fitted either directly to the function $f(\nu)$ from relation (1), resulting in a resonance peak near $b w / 2$ (see figure 2) -or the frequency shift induced by the DSFM-DU may be "undone" by adding the reference frequency to the frequency of the power spectra $u_{x}(\nu)$ and $u_{y}(\nu)$ in order to fit these 
back-shifted power spectra $u_{x}\left(\nu+\nu_{r}\right)$ and $u_{y}\left(\nu+\nu_{r}\right)$,

- Option 1: $u_{x}(t)$ raw data $\rightarrow$ FT and PSD $\rightarrow\left|u_{x}(\nu)\right|^{2} \rightarrow$ fit $\rightarrow\left\{\mathrm{e}_{\mathrm{th}}, \mathrm{Q}, \nu_{0}, \mathrm{e}_{\mathrm{n}}\right\}$

- Option 2: $u_{x}(t)$ raw data $\rightarrow$ FT and PSD $\rightarrow\left|u_{x}(\nu)\right|^{2} \rightarrow$ freq. shift $\rightarrow\left|u_{x}\left(\nu+\nu_{r}\right)\right|^{2} \rightarrow$ fit $\rightarrow\left\{e_{t h}, Q, \nu_{0}, e_{n}\right\}$

The second option is the correct one, as relation (2) holds for the "true" deflection signal with the thermal noise peak at the "correct" resonance frequency $\nu_{0}$.

We therefore fit the frequency shifted spectrum $\left|u_{x}\left(\nu+\nu_{r}\right)\right|^{2}$ to the "Lorentzian + offset" function $f(\nu)$ from relation (1), in order to obtain the "strength" $e_{t h}$ of the Lorentz function, the quality factor $Q$, the resonance frequency $\nu_{0}$ and the noise power $e_{n}$ that is not Lorentzian. For the experimental spectra of the output $u_{d}(t)$, relation (2) is rewritten as

$$
\begin{gathered}
\frac{1}{2} k T=\frac{c}{2}\left\langle z_{t h}^{2}(t)\right\rangle_{\mathrm{rms}}=\frac{c}{2} \int d \nu z_{t h}^{2}|g(\nu)|^{2}=\frac{c}{2} \int d \nu\left(e_{t h}^{d} \beta^{d}\right)^{2}|g(\nu)|^{2}= \\
=\frac{c}{2} Q \nu_{0} \frac{\pi}{2}\left(e_{t h}^{d} \beta^{d}\right)^{2} \Rightarrow \beta^{d}=\frac{1}{e_{t h}^{d}} \sqrt{\frac{2 k T}{\pi c Q \nu_{0}}}
\end{gathered}
$$

where $\beta^{d}$ (unit: $\mathrm{nm} /$ Volts) is the factor that converts the deflection signal $u_{d}(t)$ (in Volts) to the physical amplitude $z(t)$ (in nm): $z(t)=\beta^{d} u_{d}(t)$. Similarly, $\beta^{x}$ and $\beta^{y}$ convert the signals $u_{x}(t)$ and $u_{y}(t)$ into the in-phase and out-of-phase components of cantilever deflection. For the outputs $u_{x}(t)$ (and $u_{y}(t)$ ) relation $(2)$ is

$$
\begin{aligned}
& \frac{1}{2} k T=\frac{c}{2} \int d \nu\left(\left(e_{t h}^{x} \beta^{x}\right)^{2}+\left(e_{t h}^{y} \beta^{y}\right)^{2}\right)\left|g\left(\nu+\nu_{r}\right)\right|^{2}= \\
& =2 \frac{c}{2} Q \nu_{0} \frac{\pi}{2}\left(e_{t h}^{y} \beta^{y}\right)^{2} \Rightarrow \beta^{y}=\frac{1}{\sqrt{2} e_{t h}^{y}} \sqrt{\frac{2 k T}{\pi c Q \nu_{0}}}
\end{aligned}
$$

The last relation for $\beta^{y}$ in (7) is obtained because in our case the amplification of the two outputs $u_{x}(t)$ and $u_{y}(t)$ is the same; therefore, as discussed above, $\beta^{x}=\beta^{y}$. The parameters $Q, \nu_{0}$ and $e_{t h}$ are obtained from the fits, $k T$ is the temperature of the cantilever, and $c=1.6 \mathrm{~N} / \mathrm{m}$ is the force constant, determined by Sader's method [23] from the $Q$ factor and the resonance frequency. The right columns of table I (termed "second procedure") summarize the results obtained from this second calibration procedure when applied to the deflection data $u_{d}(t)$ and to the out-ofphase output $u_{y}(t)$ of the DSFM-DU. We will now comment the different fields of this table. The Lock-In amplifier is used to measure the noise density, and does not acquire time domain data, therefore the first procedure cannot be applied, and the corresponding fields are empty. The Spectrum Analyzer used allows to simultaneously measure the real-time data, as well as the 
spectrum of this data, therefore the first and second procedure can be applied. For this cantilever with a relatively large thermal noise (about $50 \mathrm{pm}$ ) the first and second calibration procedure give similar results, although the second procedure seems to have less error (see discussion below). In order to compare the results obtained from the deflection signal with that of the output(s) of the DSFM-DU a normalized calibration factor $\beta_{N}$ has been introduced that takes into account the internal gain of the DSFM-DU. This normalized calibration factor should be the same for the deflection data and the outputs of the DSFM-DU, which is indeed the case within the experimental error of the measurements.

Figure 3 shows the spectra of the deflection sensor and the outputs of the DSFM-DU for a hard cantilever ( $c=67 \mathrm{~N} / \mathrm{m}$ as determined by Sader's method [23]), with a quite low thermal noise amplitude $\left(z_{t h}=\sqrt{k T / c} \simeq 8 \mathrm{pm}\right)$. In this case, data is shown for different gains of the DSFM-DU, and the spectra have been acquired directly from the (calculated) power spectrum of the time domain signals acquired by the SFM-control unit (more precisely: from $u_{y}(t)$-images acquired with the tip far from the surface at maximum adquisition speed, without scanning and no excitation applied to the driving piezo of the cantilever). Note that for hard cantilevers, the resonance frequency may be easily outside the acquisition bandwidth of the analog to digital converters of the SFM-acquisition electronics (usually 16 bits or more and thus rather slow) and therefore the corresponding thermal noise of cantilever motion cannot be "directly seen" in the digitalized normal force (deflection) signal $u_{d}(t)$. By contrast, the thermal noise peak is easily brought into the bandwidth of the analog to digital converters when the outputs $u_{x}(t)$ and $u_{y}(t)$ are used, since this noise is now at the much lower difference frequency. For this hard cantilever, the very small thermal noise signal is usually significantly smaller than the electronic noise or other non-thermal fluctuations. In our setup, this is indeed the case for the direct deflection signal as well as for the outputs of the DSFM-DU when its internal gain is smaller than 10 (see the "scatterplots" in the inset of figure 3). Then, it is essential to "normalize" the measured signal by subtracting the electronic noise (laser off) from the total noise (laser on) as discussed above (see relation (4)). Still, for low gains this procedure does not give satisfactory results; then only the second procedure method is precise. Surprisingly the second procedure still works for gains as small as $g=1$ and for the direct deflection signal. Note that for these low gains almost all noise is electronic noise. The fit to the function $f(\nu)=e_{t h}^{2}|g(\nu)|^{2}+e_{n}^{2}$ is thus a very effective way of "filtering" all non-thermal noise. For high gains the calibration factors rise by an amount that is not compatible with the error of our measurements for both procedures. We believe that this is not a problem of the calibration procedures, instead we think that this is due to low-pass filtering 
of our DSFM-DU that reduces the (nominal) signal strength and leads to a higher calibration value at high frequency (more nanometers of deflection are "needed" for $1 \mathrm{~V}$ signal).

\section{CONCLUSION}

We have presented two methods for calibration of the oscillation amplitude in DFSM, one based on simple calculation of the RMS value of the output of the DSFM-DU $u_{y}(t)$, and the other one based on analysis of the corresponding spectrum $u_{y}(\nu)$ and calculation of the parameters $Q, \nu_{0}$, $e_{t h}$ and $e_{n}$. From the results summarized in tables I and II we conclude that both methods give consistent results, even though the second method is considerably more precise and robust, in particular for the case of hard cantilevers, where thermal noise has a much smaller amplitude and the signal to noise ratio of thermal noise (which in this case is "good" signal) vs. other noise sources is much lower. The second method has several advantages: first, it explicitly "filters" thermal noise from other noise sources, since it will only take into account signal that has a Lorentzian shape, signal not compatible with this shape is taken into account by the constant factor $e_{n}$ and the corresponding signal power is rejected for the calculation of the amplitude sensitivity. Also, fitting of a spectrum with many data points in order to obtain the four parameters $Q, \nu_{0}, e_{t h}$ and $e_{n}$ results in effective data averaging, and thus additional improvement of the estimation of the thermal noise power. And finally, from a strictly theoretical point of view, the first method is not correct because it is only an approximation valid for high $Q$ factors. In fact, the first method only "sees" thermal noise in the small bandwidth $1 / \tau \ll \nu_{0}$ around the resonance peak, but not all the noise under the Lorentz function, and in particular not the noise in the low frequency "tail" (from DC to $\left.\nu_{0}-b w / 2\right)$. This noise is "filtered away" by the DSFM-DU and thus not taken into account; therefore the first method underestimates noise, and overestimates the amplitude sensitivity (see equation (2)). Since the relation of thermal noise in the resonance peak to that in the low frequency "tail" is $Q: 1$ (see for example [15], section 3) this error is negligible for experiments in air and vacuum, but is expected to be significant for the low $Q$ factors encountered in liquids. Finally, we note that determination of the parameters $e_{t h}, Q$ and $\nu_{0}$ using the outputs of the DSFM-DU involves significant improvement of signal because of the internal gains of the DSFM-DU and the principle of Lock-in detection, which implies an important reduction of bandwidth. 


\section{ACKNOWLEDGMENTS}

The authors acknowledge stimulating discussions with Ignacio Horcas, Arvin Raman, Mariano Cuenca, Prashant Kulshreshtha and Luis Colchero. This work was supported by the Spanish Ministry of Science and Technology as well as the European Union (FEDE founds) through the projects MAT2010-21267-C02-01 and CONSOLIDER Programme "Force for Future" (CSD201000024) as well as by the "Comunidad Autónoma de la Región de Murcia" through the project "Células solares orgánicas: de la estructura molecular y nanométrica a dispositivos operativos macroscópicos". JFGM and INC acknowledge doctoral grants to the "Ministerio de Eduación" (FPU programme and "Fundación Séneca" of the "Comunidad Autónoma de la Región de Murcia", respectively).

\section{Appendix A: Processing of thermal noise by a Dynamic Unit}

We recall that a typical electronics used for analyzing the dynamics of the cantilever (see figure 1), may be implemented using a Lock-in detection scheme [1, 2, 15]: the input signal $u_{d}(t)$ to be analyzed is multiplied by two reference signals in quadrature $\left(a_{r} \cos \left(2 \pi \nu_{r} t\right)\right.$ and $\left.a_{r} \sin \left(2 \pi \nu_{r} t\right)\right)$ and then filtered with an appropriate time constant $\tau$. The corresponding output signals of the DSFM-DU are the in-phase ("phase") and the out-of-phase ("amplitude") signals ${ }^{1} u_{x}(t)$ and $u_{y}(t)$. Generally in DSFM the reference signal $a_{r} \cos \left(2 \pi \nu_{r} t\right)$ is used to excite the cantilever at resonance, then $u_{x}=0$; and changes of $u_{x}(t)$ are proportional to the phase (and thus frequency) variation of the cantilever oscillation and $u_{y}(t)$ is its oscillation amplitude. For FM-DSFM a feedback loop (Phase Locked Loop, PLL) is used to track the resonance frequency of the tipsample system by changing the excitation (i.e., the reference) frequency, in order to keep $u_{x}(t)=0$ (not shown in figure 1). In a recent work [15] we have analyzed in detail how such a DSFM-DU processes signals in the presence of thermal noise. In particular, it was shown that the DSFM-DU "frequency-shifts" a signal $u_{d}(t)=\operatorname{Re}\left[a(\nu) e^{2 \pi i \nu t}\right]$ with

$$
a(\nu)=x(\nu)+i y(\nu)=\frac{a_{0}}{1-\left(\nu / \nu_{0}\right)^{2}+i\left(\nu / \nu_{0}\right) / Q}=a_{0} g(\nu)
$$

at its input, to the sum and difference frequencies, where $g(\nu)$ is the complex "mechanical gain" of the cantilever. The outputs "phase" and "amplitude" have frequency components at $\nu_{\Sigma}=\nu_{0}+\nu_{r}$ and $\nu_{\Delta}=\nu_{0}-\nu_{r}: u_{x}(t)=\operatorname{Re}[M(t)]$ and $u_{y}(t)=\operatorname{Im}[M(t)]$ with

$$
M(t)=\frac{a(\nu)}{2}\left[\frac{1}{1+i 2 \pi \nu_{\Sigma} \tau} e^{-2 \pi i\left(t \nu_{\Sigma}+1 / 2\right)}+\frac{1}{1+i 2 \pi \nu_{\Delta} \tau} e^{+2 \pi i\left(t \nu_{\Delta}+1 / 2\right)}\right]
$$

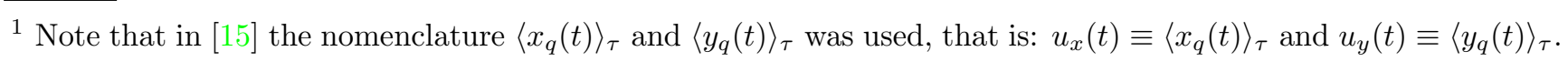


where, the matrix notation in [15], appendix A, has been translated into the more compact complex notation. When the input signal is not at a well defined frequency, but distributed around a central frequency $\nu_{0}, u_{d}(t)=\int d \nu u_{d}(\nu) e^{i 2 \pi \nu t}$, then the outputs of the DSFM-DU will be:

$$
u_{x}(t)=\frac{1}{2} \operatorname{Re}[D(t)] \text { and } u_{y}(t)=\frac{1}{2} \operatorname{Im}[D(t)]
$$

with

$$
D(t)=\int d \nu_{\Delta} d\left(\nu_{\mathrm{ref}}+\nu_{\Delta}\right) e^{2 \pi i \nu_{\Delta} t} \frac{1}{1+i 2 \pi \nu_{\Delta} \tau}
$$

where we have assumed that the term with the sum frequency $\nu_{\Sigma}$ (see relation (A2)) can be neglected because the time constant $\tau$ of the DSFM-DU is much larger than the time $1 / \nu_{\Sigma}$ (see also figure 1). The DSFM-DU therefore shifts the spectrum of its input signal $u_{d}(t)$ centered at $\nu_{0}$ to a frequency $\nu_{\Delta}=\nu_{0}-\nu_{r}$ of the output signals $u_{x}(t)$ and $u_{y}(t)$. That is, if the power spectrum of $u_{d}(t)$ has a maximum at $\nu_{0}$, the output signals $u_{x}(t)$ and $u_{y}(t)$ will have maxima at the much lower frequency $\nu_{\Delta}=\nu_{0}-\nu_{r}$, as stated in the main text of the present work. 


\section{Appendix B: Figures and Tables}

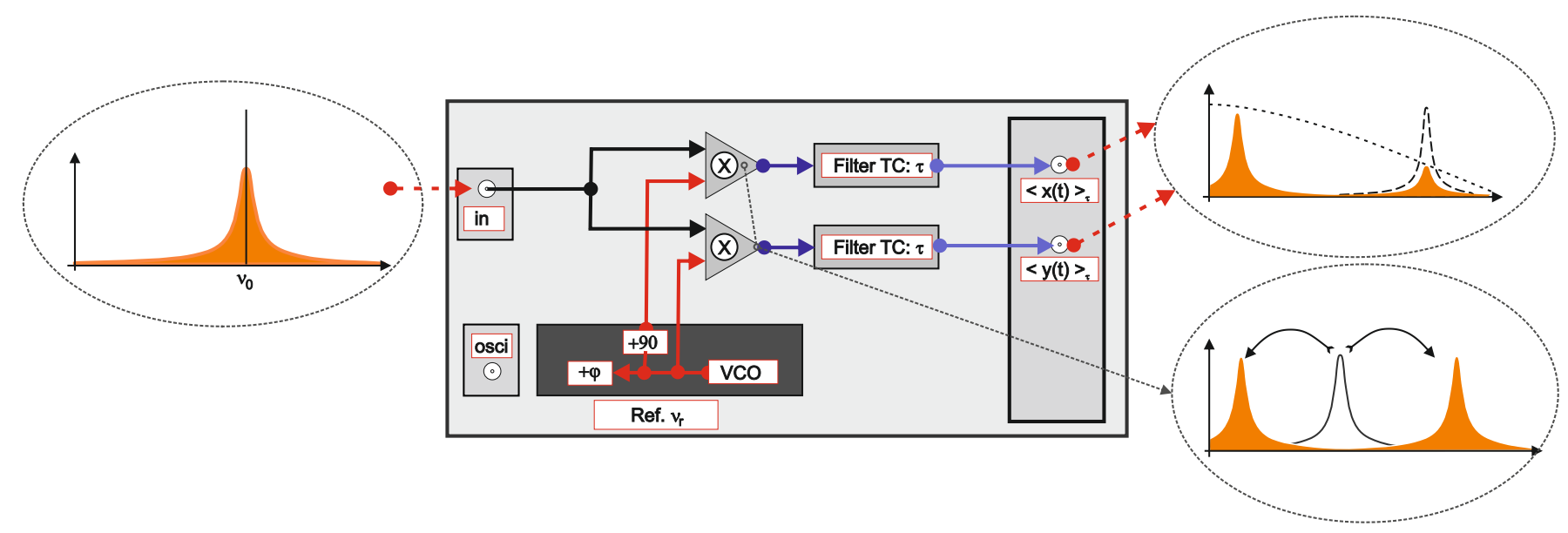

Figure 1. Schematic description of a typical Lock-in type DSFM detection unit. The signal to be analyzed by the DSFM detection is assumed to be centered around some frequency $\nu_{0}$. It enters the detection unit at the input "in", is amplified by some factor $g$ that can be generally selected and usually high-pass filtered (for simplicity the corresponding components are not shown) before it is multiplied with two reference signals in quadrature at some frequency $\nu_{\text {ref. }}$. After this multiplication, the signal is shifted to the frequencies $\nu_{0}-\nu_{\text {ref }}$ and $\nu_{0}+\nu_{\text {ref }}$. The resulting signals are then low-pass filtered to remove the higher frequency component $\left(\nu_{0}+\nu_{\text {ref }}\right)$, and brought to the outputs $u_{x}(t)$ and $u_{y}(t)$. 

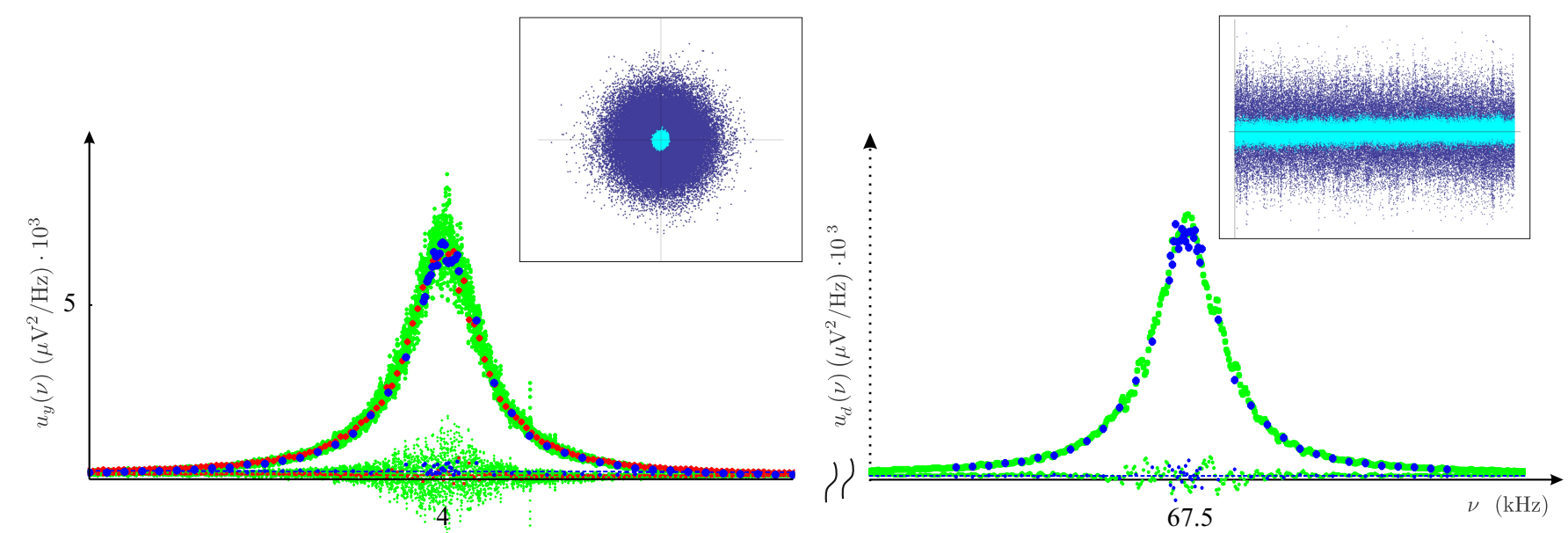

Figure 2. Power spectra $u_{y}(\nu)$ (left curve) and $u_{d}(\nu)$ (right curve; this curve is amplified to match the vertical range of the amplitude data) of the outputs $u_{y}(t)$ of the DFSM-DU unit and the deflection signal $u_{d}(t)$. Data has been acquired with an audio analyzer (green, small points), directly by the AD-converters of our control electronics (red, larger points) and with a Lock-In amplifier (blue, the largest points). Left insets: phase vs. amplitude, $\left(u_{x}(t), u_{y}(t)\right)$ data acquired with an audio analyzer. Right inset: deflection vs. time signal, $u_{d}(t)$. For both insets: dark blue, signals with the laser on; light blue, signals with the laser off. 

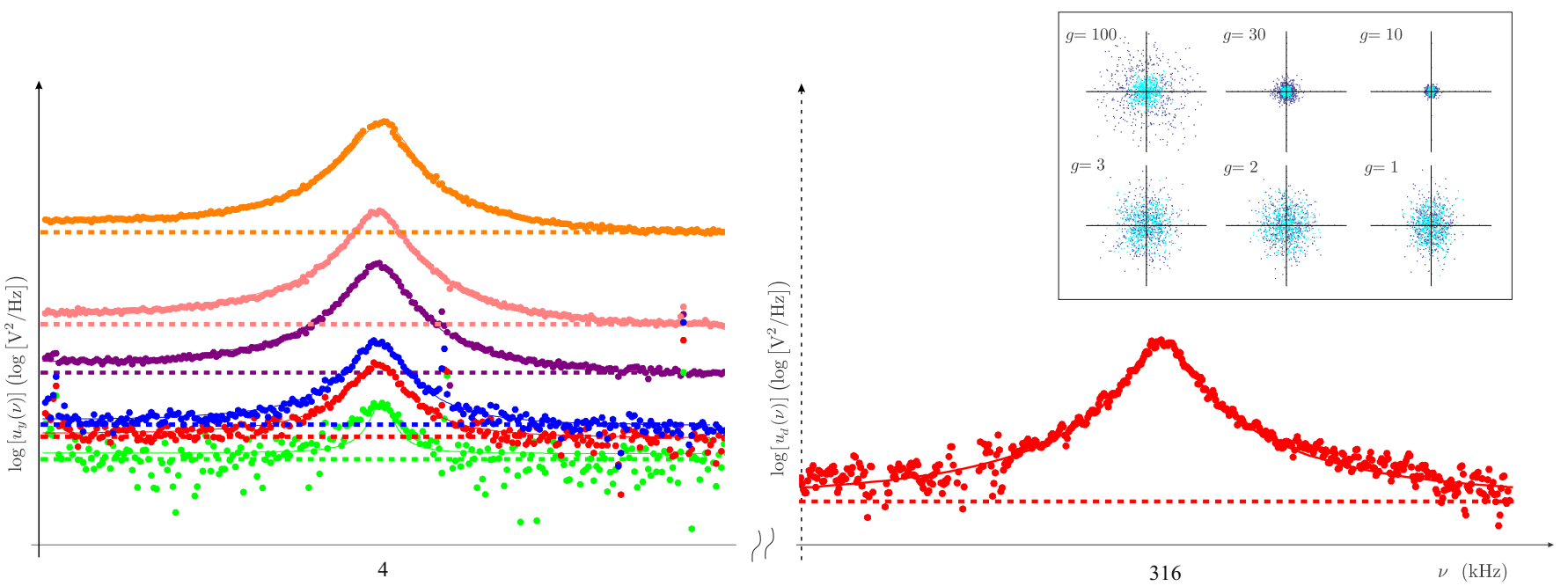

Figure 3. Thermal noise data acquired for a hard cantilever $(c=67 \mathrm{~N} / \mathrm{m})$. Left side: power spectra of the outputs $u_{y}(t)$ of the DFSM-DU unit acquired with different input gains. (a) Power spectra for gains $g=1,2,3,10,30$ and 100 . Note that in this figure a logarithmic representation of the spectra has been choosen in order to compress the vertical scale to fit all spectra with very different gains. Lower right graph: Spectrum of the deflection vs. time signal $u_{d}(t)$, measured with a digital oscilloscope. Inset: phase vs. amplitude scatter plots $\left(u_{x}(t), u_{y}(t)\right)$ from the data that was used to compute the spectra $u_{y}(\nu)$ shown left; dark blue: signals with the laser on, light blue: signals with the laser off. The upper three scatter plots of the graph correspond to $\left(u_{x}(t), u_{y}(t)\right)$ data for gains $g=100,30$ and 10; the lower three scatter plots to data for gains $g=3,2$ and 1 . 


\begin{tabular}{|c|c|c|c|c|c|c|c|c|c|c|c|}
\hline & & \multicolumn{4}{|c|}{ First Procedure } & \multicolumn{6}{|c|}{ Second Procedure } \\
\hline & & $u_{\mathrm{on}}^{\mathrm{rms}}$ & $u_{\text {off }}^{\mathrm{rms}}$ & $u_{\mathrm{th}}^{\mathrm{rms}}$ & $\beta^{\text {rms }}$ & $\nu$ & $Q$ & $e_{\mathrm{th}}$ & $e_{n}$ & $\beta_{10}^{\mathrm{PSD}}$ & $\beta_{N}^{\mathrm{PSD}}$ \\
\hline & Units $\rightarrow$ & $\mathrm{mV}$ & $\mathrm{mV}$ & $\mathrm{mV}$ & $\mathrm{nm} / \mathrm{V}$ & $\mathrm{Hz}$ & & $\mathrm{V} / \sqrt{\mathrm{Hz}} \cdot 10^{-6}$ & $\mathrm{~V} / \sqrt{\mathrm{Hz}} \cdot 10^{-6}$ & $\mathrm{~nm} / \mathrm{V}$ & $\mathrm{nm} / \mathrm{V}$ \\
\hline DSP & $\left\langle u_{y}(t)\right\rangle$ & 5.38 & 0.72 & 5.33 & 9.68 & $67438.0( \pm 14 \mathrm{ppm})$ & $138.6( \pm 0.6 \%)$ & $1.3( \pm 0.5 \%)$ & $30( \pm 1.9 \%)$ & $10.39( \pm 0.8 \%)$ & $36.7( \pm 0.8 \%)$ \\
\hline \multirow{2}{*}{ LI } & $u_{d}(t)$ & - & - & - & - & $67440( \pm 40 \mathrm{ppm})$ & $137( \pm 2.4 \%)$ & $0.35( \pm 2.4 \%)$ & $3( \pm 66 \%)$ & - & $38.7( \pm 3.6 \%)$ \\
\hline & $\left\langle u_{y}(t)\right\rangle$ & - & - & - & - & $67440( \pm 30 \mathrm{ppm})$ & $139( \pm 1.6 \%)$ & $1.3( \pm 1.6 \%)$ & $20( \pm 13 \%)$ & $10.35( \pm 1.1 \%)$ & $36.6( \pm 2.4 \%)$ \\
\hline \multirow{2}{*}{ AA } & $u_{d}(t)$ & 1.77 & 0.39 & 1.72 & 29.9 & $67441( \pm 160 \mathrm{ppm})$ & $140( \pm 6.3 \%)$ & $0.30( \pm 5 \%)$ & $5( \pm 12 \%)$ & - & $39( \pm 8 \%)$ \\
\hline & $\left\langle u_{y}(t)\right\rangle$ & 5.40 & 0.56 & 5.37 & 9.61 & $67440( \pm 5 \mathrm{ppm})$ & $137.8( \pm 0.22 \%)$ & $1.33( \pm 0.2 \%)$ & $20( \pm 1.5 \%)$ & $10.12( \pm 0.3 \%)$ & $35.8( \pm 0.3 \%)$ \\
\hline
\end{tabular}

Table I. Results obtained with the two calibration procedures applied to the measured deflection and amplitude noise acquired from a cantilever with a force constant $c=1.6 \mathrm{~N} / \mathrm{m}$. 


\begin{tabular}{|c|c|c|c|c|c|c|c|c|c|c|c|}
\hline & & \multicolumn{5}{|c|}{ First Procedure } & \multicolumn{5}{|c|}{ Second Procedure } \\
\hline & & $u_{\mathrm{on}}^{\mathrm{rms}}$ & $u_{\mathrm{off}}^{\mathrm{rms}}$ & $u_{\mathrm{th}}^{\mathrm{rms}}$ & $\beta^{\mathrm{rms}}$ & $\beta_{N}^{\mathrm{rms}}$ & $\nu$ & $Q$ & $e_{\text {th }}$ & $\beta_{G}^{\mathrm{PSD}}$ & $\beta_{N}^{\mathrm{PSD}}$ \\
\hline & Units $\rightarrow$ & $\mathrm{mV}$ & $\mathrm{mV}$ & $\mathrm{mV}$ & $\mathrm{nm} / \mathrm{V}$ & $\mathrm{nm} / \mathrm{V}$ & $\mathrm{Hz}$ & & $\mathrm{V} / \sqrt{\mathrm{Hz}} \cdot 10^{-9}$ & $\mathrm{~nm} / \mathrm{V}$ & $\mathrm{nm} / \mathrm{V}$ \\
\hline Osci & $\left\langle u_{d}(t)\right\rangle$ & 0.77 & 0.68 & 0.36 & - & 20 & $316066( \pm 7 \mathrm{ppm})$ & $588( \pm 1.3 \%)$ & $8.6( \pm 8 \%)$ & - & $53( \pm 9 \%)$ \\
\hline \multirow{6}{*}{ DSP } & $\left\langle u_{y}(t)\right\rangle_{\mathrm{G}=1}$ & 0.314 & 0.299 & 0.095 & 80 & 30 & $316060( \pm 120 \mathrm{ppm})$ & $580( \pm 22 \%)$ & $3.2( \pm 18 \%)$ & $140( \pm 29 \%)$ & $51( \pm 29 \%)$ \\
\hline & $\left\langle u_{y}(t)\right\rangle_{\mathrm{G}=2}$ & 0.33 & 0.29 & 0.16 & 50 & 40 & $316050( \pm 73 \mathrm{ppm})$ & $570( \pm 13 \%)$ & $6.5( \pm 11 \%)$ & $72( \pm 18 \%)$ & $50( \pm 18 \%)$ \\
\hline & $\left\langle u_{y}(t)\right\rangle_{\mathrm{G}=3}$ & 0.36 & 0.30 & 0.21 & 40 & 40 & $316050( \pm 59 \mathrm{ppm})$ & $570( \pm 13 \%)$ & $9.4( \pm 9 \%)$ & $50( \pm 14 \%)$ & $51( \pm 14 \%)$ \\
\hline & $\left\langle u_{y}(t)\right\rangle_{\mathrm{G}=10}$ & 0.73 & 0.38 & 0.63 & 13 & 44 & $316054( \pm 6 \mathrm{ppm})$ & $570( \pm 1.3 \%)$ & $33( \pm 1 \%)$ & $14.1( \pm 1.7 \%)$ & $50.1( \pm 1.7 \%)$ \\
\hline & $\left\langle u_{y}(t)\right\rangle_{\mathrm{G}=30}$ & 1.53 & 0.60 & 1.41 & 5.6 & 48 & $316053( \pm 0.4 \mathrm{ppm})$ & $576( \pm 0.67 \%)$ & $74.8( \pm 0.56 \%)$ & $6.21( \pm 0.9 \%)$ & $53.2( \pm 0.9 \%)$ \\
\hline & $\left\langle u_{y}(t)\right\rangle_{\mathrm{G}=100}$ & 6.24 & 2.02 & 5.91 & 1.33 & 53 & $316055( \pm 0.3 \mathrm{ppm})$ & $563( \pm 0.6 \%)$ & $316.2( \pm 0.51 \%)$ & $1.49( \pm 0.8 \%)$ & $52.9( \pm 0.8 \%)$ \\
\hline
\end{tabular}

Table II. Results obtained with the two calibration procedures for a hard cantilever (force constant $c=67$ $\mathrm{N} / \mathrm{m})$. 


\section{REFERENCES}

[1] TR Albrecht, P Grutter, D Horne, and D Rugar. Frequency-modulation detection using high-q cantilevers for enhanced force microscope sensitivity. Journal Of Applied Physics, 69(2):668-673, JAN 151991.

[2] U Durig, O Zuger, and A Stalder. Interaction Force Detection In Scanning Probe Microscopy Methods And Applications. Journal Of Applied Physics, 72(5):1778-1798, SEP 11992.

[3] F J Giessibl. Advances in atomic force microscopy. Rev. Mod. Phys., 75:949-983, Jul 2003.

[4] R García and R Pérez. Dynamic atomic force microscopy methods. Surf. Sci. Rep., 47(6-8):197-301, 2002.

[5] JP Aime, R Boisgard, L Nony, and G Couturier. Nonlinear dynamic behavior of an oscillating tip-microlever system and contrast at the atomic scale. Physical Review Letters, 82(17):3388-3391, APR 261999.

[6] Hanna Cho, Min-Feng Yu, Alexander F. Vakakis, Lawrence A. Bergman, and D. Michael McFarland. Dynamics of microcantilever integrated with geometric nonlinearity for stable and broadband nonlinear atomic force microscopy. Surface Science, 606(17-18):L74-L78, SEP 2012.

[7] SQ Hu and A Raman. Chaos in atomic force microscopy. Physical Review Letters, 96(3), JAN 27 2006.

[8] Robert W. Stark. Bistability, higher harmonics, and chaos in AFM. Materials Today, 13(9):24-32, SEP 2010.

[9] F Jamitzky, M Stark, W Bunk, WM Heckl, and RW Stark. Chaos in dynamic atomic force microscopy. Nanotechnology, 17(7, SI):S213-S220, APR 14 2006. 8th International Conference on Non-Contact Atomic Force Microscopy, Bad Essen, GERMANY, AUG 15, 2005.

[10] Álvaro San Paulo and R García. Unifying theory of tapping-mode atomic-force microscopy. Phys. Rev. B, 66:041406, Jul 2002.

[11] JF González Martínez, I Nieto Carvajal, J Abad, and J Colchero. Nanoscale measurement of the power spectral density of surface roughness: how to solve a difficult experimental challenge. Nanoscale Research Letters, 7(1):174, 2012.

[12] G Malegori and G Ferrini. Wavelet transforms to probe long- and short-range forces by thermally excited dynamic force spectroscopy. Nanotechnology, 22(19), MAY 132011. 
[13] Standford Research. Audio analyzer. SR1 200 kHz dual-domain audio analyzer, 2010.

[14] Standford Research. Lock-in. SR830 dual channel 102 kHz digital lock-in amplifier, 2009.

[15] J Colchero, M Cuenca, JF González Martínez, J Abad, B Perez García, E Palacios-Lidón, and J Abellán. Thermal frequency noise in dynamic scanning force microscopy. J. Appl. Phys., 109(2), JAN 152011.

[16] J Colchero. Procedures in Scanning Probe Microscopy, Chapter: "Force Calibration". John Wiley \& Sons, 1998.

[17] JL Hutter and J Bechhoefer. Calibration of atomic-force microscope tips. Review Of Scientific Instruments, 64(7):1868-1873, JUL 1993.

[18] HJ Butt and M Jaschke. Calculation of thermal noise in atomic-force microscopy. Nanotechnology, 6(1):1-7, JAN 1995.

[19] NA Burnham, X Chen, CS Hodges, GA Matei, EJ Thoreson, CJ Roberts, MC Davies, and SJB Tendler. Comparison of calibration methods for atomic-force microscopy cantilevers. Nanotechnology, 14(1):1-6, JAN 2003.

[20] RS Gates, MG Reitsma, JA Kramar, and JR Pratt. Atomic force microscope cantilever flexural stiffness calibration: Toward a standard traceable method. Journal Of Research Of The National Institute Of Standards And Technology, 116(4):703-727, JUL-AUG 2011.

[21] RW Stark, T Drobek, and WM Heckl. Thermomechanical noise of a free v-shaped cantilever for atomic-force microscopy. Ultramicroscopy, 86(1-2):207-215, JAN 2001. 2nd International Conference on Scanning Probe Microscopy, Sensors, and Nanostructures, HEIDELBERG, GERMANY, MAY 28-31, 2000.

[22] J te Riet, AJ Katan, C Rankl, SW Stahl, AM van Buul, IY Phang, A Gomez-Casado, P Schon, JW Gerritsen, A Cambi, AE Rowan, GJ Vancso, P Jonkheijm, J Huskens, TH Oosterkamp, H Gaub, P Hinterdorfer, CG Figdor, and S Speller. Inter laboratory round robin on cantilever calibration for AFM force spectroscopy. Ultramicroscopy, 111(12):1659-1669, DEC 2011.

[23] JE Sader, JWM Chon, and P Mulvaney. Calibration of rectangular atomic force microscope cantilevers. Review Of Scientific Instruments, 70(10):3967-3969, OCT 1999. 